\title{
Identification of genes and pathways in esophageal adenocarcinoma using bioinformatics analysis
}

\author{
FENG HE ${ }^{1}, \mathrm{BO} \mathrm{AI}^{1}$ and LEI TIAN ${ }^{2}$ \\ Departments of ${ }^{1}$ Thoracic Surgery and ${ }^{2}$ Clinical Laboratory, Tongji Hospital, Tongji Medical College, \\ Huazhong University of Science and Technology, Wuhan, Hubei 430030, P.R. China
}

Received April 12, 2018; Accepted July 19, 2018

DOI: $10.3892 /$ br.2018.1134

\begin{abstract}
Esophageal adenocarcinoma(EAC) is one of the most common subtypes of esophageal cancer, and is associated with a low 5-year survival rate. The present study aimed to identify key genes and pathways associated with EAC using bioinformatics analysis. The gene expression profiles of GSE92396, which includes 12 EAC samples and 9 normal esophageal samples, were downloaded from the Gene Expression Omnibus database. Differentially expressed genes (DEGs) between the EAC and normal samples were identified using the limma package in R language. Gene ontology and Kyoto Encyclopedia of Genes and Genomes (KEGG) pathway enrichment analyses of the identified DEGs were conducted using the online analysis tool, the Database for Annotation, Visualization and Integrated Discovery. A protein-protein interaction (PPI) network of the DEGs was constructed using the Search Tool for the Retrieval of Interacting Genes (STRING) database and Cytoscape software. Finally, module analysis was conducted for the PPI network using the MCODE plug-in in Cytoscape. Of the 386 DEGs identified, the 150 upregulated genes were mainly enriched in the KEGG pathways of complement and
\end{abstract}

Correspondence to: Dr Feng He, Department of Thoracic Surgery, Tongji Hospital, Tongji Medical College, Huazhong University of Science and Technology, 1095 Jiefang Avenue, Wuhan, Hubei 430030, P.R. China

E-mail: drhefeng@126.com

Abbreviations: EAC, esophageal adenocarcinoma; GEO, Gene Expression Omnibus; DEGs, differentially expressed genes; PPI, protein-protein interaction; GO, gene ontology; KEGG, Kyoto Encyclopedia of Genes and Genomes; FC, fold-change; DAVID, Database for Annotation, Visualization and Integrated Discovery; IL, interleukin; IVL, involucrin; TIMP1, tissue inhibitor of metalloproteinase 1; FN1, fibronectin 1; SERPINE1, serpin family E member 1; SERPINA1, serpin family A member 1, CFTR, cystic fibrosis transmembrane conductance regulator; SPP1, secreted phosphoprotein 1; COL1A1, collagen type I alpha 1 chain; AGT, angiotensinogen; OPN, osteopontin; CXC, C-X-C motif; RAS, renin-angiotensin system

Key words: esophageal adenocarcinoma, bioinformatics analysis, differentially expressed genes, enrichment analysis, protein-protein interaction network coagulation cascades, maturity onset diabetes of the young and protein digestion and absorption; and the 236 downregulated genes were mainly enriched in amoebiasis, retinol metabolism and drug metabolism-cytochrome P450. Based on information from the STRING database, a PPI network comprising of 369 nodes and 534 edges was constructed in Cytoscape. The top 10 hub nodes with the highest degrees were determined as interleukin-8, involucrin, tissue inhibitor of metalloproteinase 1, fibronectin 1, serpin family E member 1, serpin family A member 1, cystic fibrosis transmembrane conductance regulator, secreted phosphoprotein 1, collagen type I alpha 1 chain and angiotensinogen. A total of 6 modules were detected from the PPI network that satisfied the criteria of MCODE score $>4$ and number of nodes $>4$. KEGG pathways enriched for the module DEGs were mainly within arachidonic acid metabolism, complement and coagulation cascades and rheumatoid arthritis. In conclusion, identification of these key genes and pathways may improve understanding of the mechanisms underlying the development of EAC, and may be used as diagnostic and therapeutic targets in EAC.

\section{Introduction}

Esophageal cancer is among the most common malignancies worldwide, and in the United States has a 5-year survival rate following diagnosis of only $\sim 19 \%$ (1). Squamous cell carcinoma and adenocarcinoma are the two main subtypes of esophageal cancer. The incidence of esophageal adenocarcinoma (EAC) has increased substantially in the United States, Western Europe, Australia and other developed countries over the past four decades (2). It is generally accepted that gastroesophageal reflux disease and obesity are explanations for the increased incidence of EAC (3). However, the underlying mechanism remains unclear.

Several genes have been reported to serve important roles in the development of EAC. The P53 gene has been found to be dysregulated in most cancer types (4). Furthermore, it is considered that P53 may be involved in the development of different cancers. For instance, a cohort study of chemoradiotherapy-naive surgically treated EAC reported that p53 expression was significantly correlated with disease-free survival and overall survival, independent of tumor stage (5). Meanwhile, a genome-wide association study of 2,515 EAC cases and 3,207 controls provided data to suggest that germline 
variations at the cyclin-dependent kinase inhibitor 2A locus may influence susceptibility to EAC (6). In addition, Gli and epithelial-mesenchymal transition-related protein expression was previously examined by western blot analysis in paired EAC patient tissues and cell lines. The results suggested that Gli may be critical for the metastasis and recurrence of esophageal adenocarcinomas (7). Osteopontin (OPN) isoforms have also been investigated in EAC, where results indicated that all OPN isoforms were frequently co-overexpressed in primary EACs, and that isoforms OPNb and OPNc enhanced invasion and dissemination through collective yet distinct mechanisms (8). However, despite these in-depth studies to identify novel targets for the treatment of EAC, there lacks a comprehensive presentation of the key genes and pathways implicated in EAC.

Gene expression profile analysis is a high-throughput method for detecting messenger RNA expression in tissue or cell samples. By analyzing the different gene expression between cancer patients and normal controls, an improved understanding of the molecular pathogenesis of a tumor can be obtained, facilitating the identification of the potential target genes and pathways for therapy $(9,10)$.

The present study aimed to investigate the pathogenesis of EAC by a computational bioinformatics analysis of gene expression. Data from the Gene Expression Omnibus (GEO) database was extracted, and differentially expressed genes (DEGs) between EAC and normal samples were identified. The possible functions of the DEGs were predicted using enrichment analysis. Furthermore, protein-protein interaction (PPI) networks were visualized and module analysis was conducted using Cytoscape software to search for key genes that may be involved in the development of EAC.

\section{Materials and methods}

Affymetrix microarray data. The gene expression profiles of GSE92396, contributed by Peng et al (11), were downloaded from the Gene Expression Omnibus (GEO) database (http://www.ncbi.nlm.nih.gov/geo/). The platform was GPL6244, HuGene-1_0-st Affymetrix Human Gene 1.0 ST Array. The dataset included 12 esophageal adenocarcinoma samples and 9 normal esophageal samples; 9 were tumor-normal pairs.

Identification of DEGs. The data were pre-processed in R language (version 3.4.3; https://www.r-project.org/) using the oligo package (version 1.32.0; https://www.bioconductor. org/packages/release/bioc/html/oligo.html) $(12,13)$. Probe levels were calculated and converted into the gene expression levels according to the annotation files in the GEO database. The DEGs of GSE92396 between the normal tissues and the tumor samples were analyzed with limma package (version 3.34.8) in R language (14). Fold-changes (FCs) in the gene expression values were calculated. $\mid \log _{2} \mathrm{FCl}>2$ and adjusted P-values $<0.05$ were considered to be the cut-off criteria for the identification of DEGs. A volcano plot was drawn using the gplots package (version 3.0.1).

Gene ontology $(G O)$ and pathway enrichment analysis of the DEGs. The online analysis tool, the Database for Annotation,
Visualization and Integrated Discovery (DAVID; version 6.8; http://david.abcc.ncifcrf.gov/) was used to analyse the DEGs for GO term and Kyoto Encyclopedia of Genes and Genomes (KEGG) pathway enrichment. Enriched terms with $>2$ genes and a P-value $<0.05$ were considered to be statistically significant.

Construction of PPI network and screening of modules. The online analysis tool, the Search Tool for the Retrieval of Interacting Genes (STRING version 10.0; http://string-db.org/) was used to assess the PPI network of the DEGs, with the required confidence (combined score) $>0.4$. Visualization of the network and module analysis were performed with Cytoscape software (version 3.6.0; http://www.cytoscape.org/) and the MCODE plug-in (version 1.5.1) (15). The degree was statistically analysed using the CentiScaPe plug-in (version 2.2) to obtain hub nodes or genes in the PPI network (16). An MCODE computed node score $>4$ and node number $>4$ were considered as the cut-off criteria. Subsequent GO function and KEGG pathway enrichment analyses of the DEGs in the modules were performed using DAVID.

\section{Results}

Identification of DEGs. To identify DEGs between EAC samples and normal controls, the microarray dataset GSE92396, obtained from the GEO database, was screened. DEGs with $\mid \log _{2} \mathrm{FCl}>2$ and a P-value $<0.05$ were determined. A total of 386 DEGs were identified in EAC samples compared with in the normal controls, including 150 upregulated and 236 downregulated DEGs. The volcano plot is presented in Fig. 1.

GO and pathway enrichment analysis of DEGs. To categorize the representation of DEGs and the involved pathways, GO and KEGG pathway enrichment analyses were performed using the online tool DAVID. The upregulated DEGs were enriched in 60 GO terms and 3 KEGG pathways. The GO functions enriched for the upregulated DEGs were mainly within the extracellular exosome $\left(\mathrm{P}=1.09 \times 10^{-14}\right)$, extracellular space $\left(\mathrm{P}=5.44 \times 10^{-15}\right)$ and extracellular region $\left(\mathrm{P}=1.61 \times 10^{-10}\right)$. KEGG pathways enriched for the upregulated DEGs were mainly in complement and coagulation cascades $\left(\mathrm{P}=6.84 \times 10^{-5}\right)$, maturity onset diabetes of the young $(\mathrm{P}=0.002282)$ and protein digestion and absorption $(\mathrm{P}=0.012483)$.

The downregulated DEGs were enriched in 67 GO terms and 5 KEGG pathways. The GO functions enriched for the downregulated DEGs were mainly within the extracellular exosome $\left(\mathrm{P}=8.36 \times 10^{-12}\right)$, epidermis development $\left(\mathrm{P}=3.26 \times 10^{-22}\right)$ and keratinocyte differentiation $\left(\mathrm{P}=2.62 \times 10^{-20}\right)$. KEGG pathways enriched for the downregulated DEGs were mainly in amoebiasis $\left(\mathrm{P}=3.46 \times 10^{-4}\right)$, retinol metabolism $(\mathrm{P}=0.020258)$ and drug metabolism-cytochrome $\mathrm{P} 450(\mathrm{P}=0.022809)$. The top 10 terms of the GO enrichment analysis for up- and downregulated genes are presented respectively in Table I. The results of KEGG enrichment analysis for up- and downregulated genes are presented respectively in Table II.

Construction of PPI network and screening of modules. Based on information from the STRING database, a PPI network comprising of 369 nodes and 534 edges was constructed using 
Table I. GO enrichment analysis of the differentially expressed genes.

\begin{tabular}{|c|c|c|c|}
\hline Category & Term & Count & P-value \\
\hline \multicolumn{4}{|l|}{ Upregulated genes } \\
\hline GOTERM_CC_DIRECT & GO:0005615 extracellular space & 43 & $5.44 \times 10^{-15}$ \\
\hline GOTERM_CC_DIRECT & GO:0070062 extracellular exosome & 62 & $1.09 \times 10^{-14}$ \\
\hline GOTERM_CC_DIRECT & GO:0005576 extracellular region & 40 & $1.61 \times 10^{-10}$ \\
\hline GOTERM_CC_DIRECT & GO:0005578 proteinaceous extracellular matrix & 15 & $3.09 \times 10^{-8}$ \\
\hline GOTERM_BP_DIRECT & GO:0022617 extracellular matrix disassembly & 9 & $2.36 \times 10^{-7}$ \\
\hline GOTERM_CC_DIRECT & GO:0031012 extracellular matrix & 14 & $7.17 \times 10^{-7}$ \\
\hline GOTERM_CC_DIRECT & GO:0016324 apical plasma membrane & 13 & $3.74 \times 10^{-6}$ \\
\hline GOTERM_BP_DIRECT & GO:0030198 extracellular matrix organization & 11 & $6.13 \times 10^{-6}$ \\
\hline GOTERM_CC_DIRECT & GO:0005796 Golgi lumen & 8 & $1.15 \times 10^{-5}$ \\
\hline GOTERM_BP_DIRECT & GO:0030574 collagen catabolic process & 7 & $1.58 \times 10^{-5}$ \\
\hline \multicolumn{4}{|l|}{ Downregulated genes } \\
\hline GOTERM_BP_DIRECT & GO:0008544 epidermis development & 22 & $3.26 \times 10^{-22}$ \\
\hline GOTERM_BP_DIRECT & GO:0030216 keratinocyte differentiation & 20 & $2.62 \times 10^{-20}$ \\
\hline GOTERM_CC_DIRECT & GO:0001533 cornified envelope & 15 & $1.04 \times 10^{-16}$ \\
\hline GOTERM_BP_DIRECT & GO:0018149 peptide cross-linking & 14 & $1.69 \times 10^{-14}$ \\
\hline GOTERM_BP_DIRECT & GO:0031424 keratinization & 13 & $2.97 \times 10^{-13}$ \\
\hline GOTERM_MF_DIRECT & GO:0005198 structural molecule activity & 21 & $7.55 \times 10^{-12}$ \\
\hline GOTERM_CC_DIRECT & GO:0070062 extracellular exosome & 74 & $8.36 \times 10^{-12}$ \\
\hline GOTERM_CC_DIRECT & GO:0030057 desmosome & 8 & $8.15 \times 10^{-9}$ \\
\hline GOTERM_MF_DIRECT & GO:0004867 serine-type endopeptidase inhibitor activity & 10 & $1.66 \times 10^{-6}$ \\
\hline GOTERM_BP_DIRECT & GO:0061436 establishment of skin barrier & 6 & $1.76 \times 10^{-6}$ \\
\hline
\end{tabular}

GO, gene ontology;_BP, biological process;_CC, cellular component; _MF, molecular function.

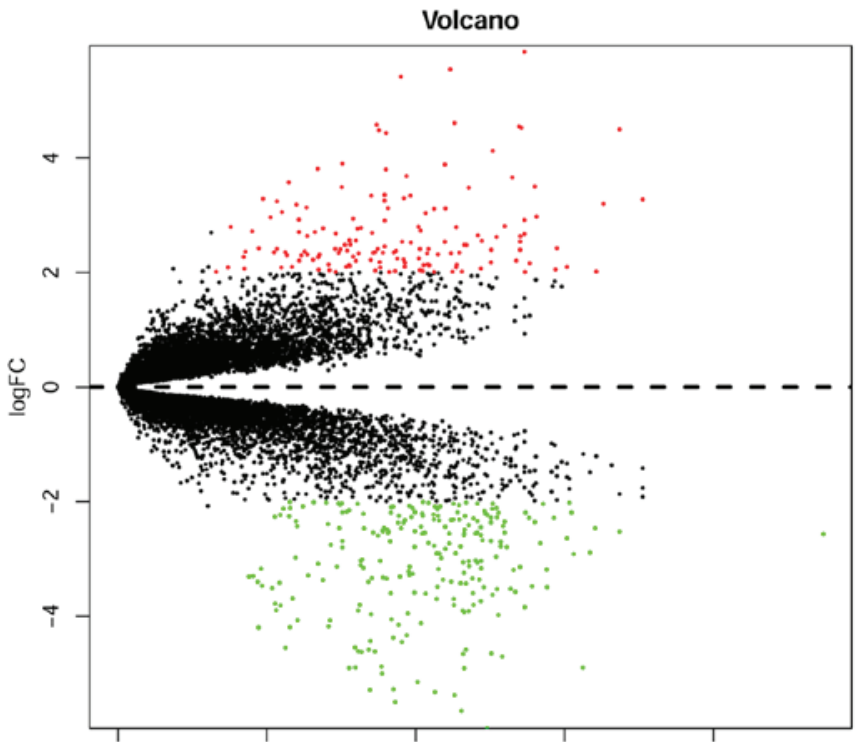

Figure 1. Volcano plot of differentially expressed genes in esophageal adenocarcinoma. Black, non-differentially expressed genes; red, significantly upregulated genes; green, significantly downregulated genes (based on $\mid \log _{2} \mathrm{FCl}>2$ and adjusted $\left.\mathrm{P}<0.05\right)$. FC, fold-change; adj.P.Val, adjusted P-value.

the Cytoscape software (Fig. 2). The top 10 hub nodes with the highest degrees were interleukin (IL)-8, involucrin (IVL), tissue inhibitor of metalloproteinase 1 (TIMP1), fibronectin 1
(FN1), serpin family E member 1 (SERPINE1), serpin family A member 1 (SERPINA1), cystic fibrosis transmembrane conductance regulator (CFTR), secreted phosphoprotein 1 (SPP1), collagen type I alpha 1 chain (COL1A1) and angiotensinogen (AGT). A total of 6 modules from the PPI network satisfied the criteria of an MCODE computed node score $>4$ and number of nodes $>4$. The results are presented in Fig. 3. The functional annotation of the DEGs involved in the modules was determined using DAVID. The results showed that the module DEGs were enriched in 66 GO terms and 9 KEGG pathways. The GO functions enriched for the module DEGs were mainly within the extracellular exosome $\left(\mathrm{P}=1.27 \times 10^{-8}\right)$, extracellular region $\left(\mathrm{P}=8.63 \times 10^{-11}\right)$ and extracellular space $\left(\mathrm{P}=1.21 \times 10^{-7}\right)$. KEGG pathways enriched for the module DEGs were mainly within arachidonic acid metabolism $\left(\mathrm{P}=1.02 \times 10^{-}\right.$ $\left.{ }^{4}\right)$, complement and coagulation cascades $\left(\mathrm{P}=1.56 \times 10^{-4}\right)$, and rheumatoid arthritis $\left(\mathrm{P}=3.98 \times 10^{-4}\right)$. The top 10 terms of the GO and KEGG enrichment analyses for module DEGs are presented in Table III.

\section{Discussion}

EAC is one of the most common subtypes of esophageal cancer (17), and only $\sim 19 \%$ of patients survive 5 year after diagnosis in the United States (1). Therefore, there is a need to screen for key genes and pathways that are associated with the progression of EAC, with the aim of improving its diagnosis and treatment. 


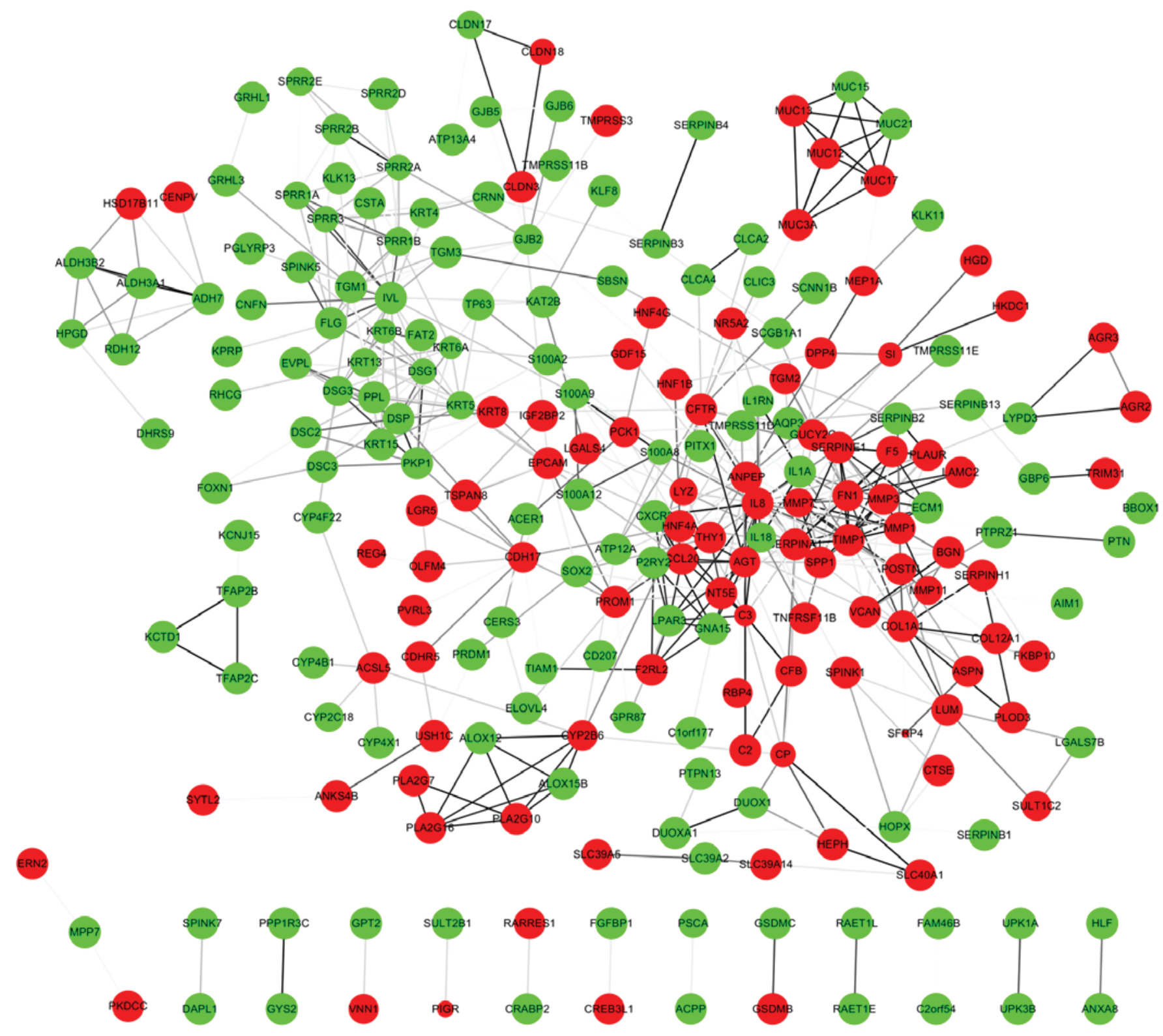

Figure 2. Protein-protein interaction network of differentially expressed genes. Red, significantly upregulated genes; green, significantly downregulated genes Node size is negatively related to P-value; edge color and width are positively related to combined score.

The present study used bioinformatics analysis to identify the DEGs between EAC and normal tissue expression profiles. The results revealed that the expression of 386 genes was significantly altered in EAC samples (150 upregulated and 236 downregulated genes) compared with in the normal controls. A PPI network was constructed to reveal the associations between these genes. The top 10 genes with the highest degrees were identified. Furthermore, 6 modules were selected according to their respective MCODE computed node scores $(>4)$, and their functions were determined by GO and KEGG pathway analyses.

The GO functions enriched for the upregulated DEGs were mainly within the extracellular exosome, extracellular space and extracellular region. KEGG pathways enriched for the upregulated DEGs were mainly within complement and coagulation cascades, maturity onset diabetes of the young and protein digestion and absorption. The GO functions enriched for the downregulated DEGs were mainly within the extracellular exosome, epidermis development and keratinocyte differentiation. KEGG pathways enriched for the downregulated DEGs were mainly within amoebiasis, retinol metabolism and drug metabolism-cytochrome P450. Previous study has demonstrated that activation of the coagulation cascade affected tumor development (18). The underlying mechanism through which coagulation cascade proteins promote tumorigenesis remains unclear. Therefore, investigating these identified signaling pathways may aid to elucidate the carcinogenic mechanism behind EAC.

Based on the results of PPI network construction for the DEGs, a number of hub nodes were identified. The top 10 hub 
Table II. KEGG pathway enrichment analysis of the differentially expressed genes.

\begin{tabular}{llrr}
\hline Category & \multicolumn{1}{c}{ Term } & Count & P-value \\
\hline Upregulated genes & & & 7 \\
KEGG_PATHWAY & hsa04610 Complement and coagulation cascades & 4 & $6.84 \times 10^{-5}$ \\
KEGG_PATHWAY & hsa04950 Maturity onset diabetes of the young & 5 & 0.002282 \\
KEGG_PATHWAY & hsa04974 Protein digestion and absorption & 0.012483 \\
Downregulated genes & & 7 & $3.46 \times 10^{-4}$ \\
KEGG_PATHWAY & hsa05146 Amoebiasis & 4 & 0.020258 \\
KEGG_PATHWAY & hsa00830 Retinol metabolism & 4 & 0.022809 \\
KEGG_PATHWAY & hsa00982 Drug metabolism - cytochrome P450 & 4 & 0.034682 \\
KEGG_PATHWAY & hsa05204 Chemical carcinogenesis & 3 & 0.038991 \\
KEGG_PATHWAY & hsa00350 Tyrosine metabolism & &
\end{tabular}

KEGG, Kyoto Encyclopedia of Genes and Genomes; hsa, homo sapiens.
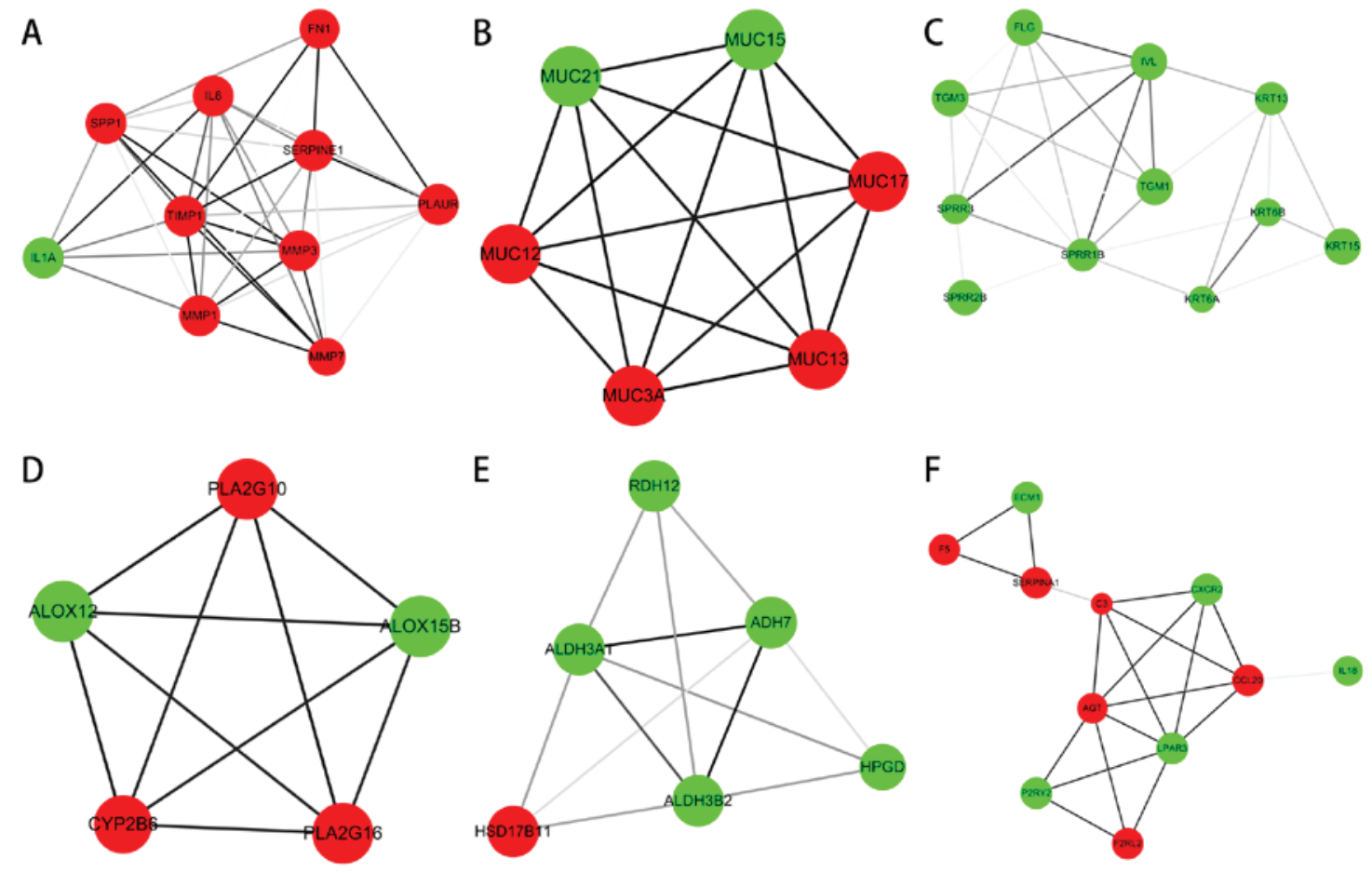

Figure 3. (A-F) Modules of PPI network determined using the MCODE plugin of Cytoscape. Red, significantly upregulated genes; green, significantly downregulated genes. Node size is negatively related to P-value; edge color and width are positively related to combined score.

nodes with the highest degrees were IL8, IVL, TIMP1, FN1, SERPINE1, SERPINA1, CFTR, SPP1, COL1A1 and AGT. IL8, also named C-X-C motif (CXC) chemokine ligand 8, is a chemokine that mainly attracts inflammatory leukocyte infiltrate by acting on CXC chemokine receptor $1 / 2$. Recent speculations propose that IL8 serves important roles in angiogenesis and survival signaling for cancer stem cells, and that the interleukin may stimulate the secretion of local growth factors in malignant tumors (19). IL8 stimulation on endothelial cells has been reported to begin angiogenic processes characterized by secretion of matrix metalloproteinases (MMPs), which can break down the extracellular matrix and stimulate the formation of new vessels (20). One study reported that IL8 was significantly upregulated in esophageal carcinogenesis, being detected in the serum of patients with esophageal adenocarcinoma (21). IVL is a squamous cell differentiation marker, and is associated with terminal differentiation of epithelial cells $(22,23)$. Upon IL4 stimulation, the overall esophageal epithelia still contained stratified morphology. However, IVL was significantly decreased in esophageal basal and suprabasal layers, which was associated with a disorganized morphology of stratified layers on the basal side (24). TIMP1 is an inhibitor of matrix metalloproteinases, which has a key role in cancer cell dissemination and endothelial cell migration in angiogenesis (25). High serum levels of TIMP1 have been associated with tumor progression and poor prognosis in esophageal cancer patients (26). FN1, a mesenchymal marker (27), is an extracellular matrix glycoprotein that serves 
Table III. GO and KEGG enrichment analyses of the differentially expressed genes in the modules.

\begin{tabular}{llrl}
\hline Category & \multicolumn{1}{c}{ Term } & Count & P-value \\
\hline GOTERM_CC_DIRECT & GO:0005576 extracellular region & 22 & $8.63 \times 10^{-11}$ \\
GOTERM_BP_DIRECT & GO:0018149 peptide cross-linking & 7 & $5.00 \times 10^{-9}$ \\
GOTERM_CC_DIRECT & GO:0070062 extracellular exosome & 25 & $1.27 \times 10^{-8}$ \\
GOTERM_BP_DIRECT & GO:0030216 keratinocyte differentiation & 7 & $6.52 \times 10^{-8}$ \\
GOTERM_CC_DIRECT & GO:0005615 extracellular space & 17 & $1.21 \times 10^{-7}$ \\
GOTERM_BP_DIRECT & GO:0031424 keratinization & 6 & $2.16 \times 10^{-7}$ \\
GOTERM_BP_DIRECT & GO:0016266 O-glycan processing & 6 & $6.72 \times 10^{-7}$ \\
GOTERM_BP_DIRECT & GO:0022617 extracellular matrix disassembly & 6 & $2.20 \times 10^{-6}$ \\
GOTERM_MF_DIRECT & GO:0005198 structural molecule activity & 8 & $3.19 \times 10^{-6}$ \\
GOTERM_CC_DIRECT & GO:0005796 Golgi lumen & 6 & $4.70 \times 10^{-6}$ \\
KEGG_PATHWAY & hsa00590 Arachidonic acid metabolism & 5 & $1.02 \times 10^{-4}$ \\
KEGG_PATHWAY & hsa04610 Complement and coagulation cascades & 5 & $1.56 \times 10^{-4}$ \\
KEGG_PATHWAY & hsa05323 Rheumatoid arthritis & 5 & $3.98 \times 10^{-4}$ \\
KEGG_PATHWAY & hsa00982 Drug metabolism - cytochrome P450 & 4 & 0.002504 \\
KEGG_PATHWAY & hsa00980 Metabolism of xenobiotics by cytochrome P450 & 4 & 0.003187 \\
KEGG_PATHWAY & hsa00350 Tyrosine metabolism & 3 & 0.008675 \\
KEGG_PATHWAY & hsa00830 Retinol metabolism & 3 & 0.028145 \\
KEGG_PATHWAY & hsa00010 Glycolysis/Gluconeogenesis & 3 & 0.02977 \\
KEGG_PATHWAY & hsa05204 Chemical carcinogenesis & 3 & 0.041201 \\
\hline
\end{tabular}

GO, gene ontology; KEGG, Kyoto Encyclopedia of Genes and Genomes; _BP, biological process;_CC, cellular component; _MF, molecular function; hsa, homo sapiens.

key roles in cell differentiation, growth and migration; through which it is associated with certain processes including wound healing, embryonic development and carcinogenesis (28). SERPINE1, also known as plasminogen activator inhibitor-1, is involved in the inhibition of urokinase-type plasminogen activator (29). It serves important roles in increasing tumor invasion and angiogenesis, and has been correlated with a poor prognosis (30). A high tumor level of plasminogen activator inhibitor-1 in patients with primary breast cancer is reportedly suggestive of poor prognosis (31), through this association requires verification in EAC. SERPINA1 encodes for $\alpha 1$-antitrypsin, which targets several proteases, including elastase, plasmin, thrombin, trypsin, chymotrypsin, and plasminogen activator (32). One study suggested that $\alpha 1$-antitrypsin may be involved in lung adenocarcinoma metastasis by targeting fibronectin (33). CFTR encodes an ATP-binding cassette membrane protein that functions as a chloride channel, and is mutated in cystic fibrosis $(34,35)$. A previous large-scale meta-analysis suggested that the novel single nucleotide polymorphism (SNP) rs17451754, which is located within intron 21 of the CFTR gene, markedly associates with the risk of Barrett's esophagus and EAC (36). This region is reportedly involved with the enhancer histone modifications in the gastrointestinal tract mucosa and DNAse hypersensitivity (37). SPP1, also known as OPN, encodes a protein that binds hydroxyapatite, and is a cytokine that upregulates interferon- $\gamma$ and IL12 (38). A previous study reported that SPP1 isoforms collectively enhanced tumor cell invasion and dissemination in EAC (8). COL1A1 encodes the pro- $\alpha 1$ chains of type I collagen. It has been reported COL1A1 is overexpressed in many tumours, including gastric cancer, hepatocellular carcinoma, non-small cell lung cancer, and colorectal cancer (39-42). One study suggested that COL1A1 may promote metastasis in colorectal cancer by regulating the Wnt/planar cell polarity pathway (39). AGT is involved in the renin-angiotensin system (RAS). Previous study reported that RAS participated in the physiological control of esophageal motor activity (43). Together with these previous findings, the present results are suggestive that RAS may be involved in the contraction disorder of esophageal adenocarcinoma.

A total of 6 modules from the PPI network satisfied the criteria of MCODE score $>4$ and number of nodes $>4$. The GO functions enriched for the module DEGs were mainly within the extracellular exosome, extracellular region and extracellular space. KEGG pathways enriched for the module DEGs were mainly in arachidonic acid metabolism, complement and coagulation cascades and rheumatoid arthritis. A previous study suggested that the activated arachidonic acid metabolism pathway serves an important role in tumorigenesis (44). The enzymes activated by this pathway and their products promote the inflammatory response and have been implicated in multiple cellular processes, including cell proliferation, invasion and metastasis, and thus may promote tumorigenesis. Additionally, previous study has demonstrated that activation of the coagulation cascade affected tumor development (18). Therefore, the arachidonic acid metabolism and complement and coagulation cascades pathways may be involved in the development of EAC.

In conclusion, the present study identified the genes differentially expressed between EAC and normal samples. 
The top most altered DEGs included IL8, IVL, TIMP1, FN1, SERPINE1, SERPINA1, CFTR, SPP1, COL1A1 and AGT, and the pathways of arachidonic acid metabolism, complement and coagulation cascades, and rheumatoid arthritis may potentially be used as diagnostic and therapeutic targets in EAC. However, the present study is limited to an extent due to the small sample size and lack of experimental validation. Further experimental confirmation of the expression profile in EAC by immunoblotting or immunohistochemical staining is therefore required to validate the current findings.

\section{Acknowledgements}

Not applicable.

\section{Funding}

Not applicable.

\section{Availability of data and materials}

The datasets used during the current study are available in the Gene Expression Omnibus database (accession no. GSE92396; https://www.ncbi.nlm.nih.gov/geo/query/acc. cgi?acc=GSE92396).

\section{Authors' contributions}

FH and BA designed the study. FH and LT analyzed and interpreted the data. FH was primarily responsible for writing the manuscript. All authors reviewed and approved the final manuscript.

\section{Ethics approval and consent to participate}

Not applicable.

\section{Consent for publication}

Not applicable.

\section{Competing interests}

The authors declare that they have no competing interests.

\section{References}

1. Siegel RL, Miller KD and Jemal A: Cancer statistics, 2016. CA Cancer J Clin 66: 7-30, 2016.

2. Reid BJ, Li X, Galipeau PC and Vaughan TL: Barrett's oesophagus and oesophageal adenocarcinoma: Time for a new synthesis. Nat Rev Cancer 10: 87-101, 2010.

3. Cowie A, Noble F and Underwood T: Strategies to improve outcomes in esophageal adenocarcinoma. Expert Rev Anticancer Ther 14: 677-687, 2014

4. Kalatskaya I: Overview of major molecular alterations during progression from Barrett's esophagus to esophageal adenocarcinoma. Ann NY Acad Sci 1381: 74-91, 2016.

5. Ten Kate FJ, Suzuki L, Dorssers LC, Dinjens WN, Jones DT, Nieboer D, Doukas M, Van Lanschot JJ, Wijnhoven BP, Looijenga LH, et al: Pattern of p53 protein expression is predictive for survival in chemoradiotherapy-naive esophageal adenocarcinoma. Oncotarget 8: 104123-104135, 2017.
6. Buas MF, Levine DM, Makar KW, Utsugi H, Onstad L, Li X, Galipeau PC, Shaheen NJ, Hardie LJ, Romero Y, et al: Integrative post-genome-wide association analysis of CDKN2A and TP53 SNPs and risk of esophageal adenocarcinoma. Carcinogenesis 35: 2740-2747, 2014.

7. Wang L, Jin JQ, Zhou Y, Tian Z, Jablons DM and He B: Gli is activated and promotes epithelial-mesenchymal transition in human esophageal adenocarcinoma. Oncotarget 9: 853-865, 2017.

8. Lin J, Myers AL, Wang Z, Nancarrow DJ, Ferrer-Torres D, Handlogten A, Leverenz K, Bao J, Thomas DG, Wang TD, et al: Osteopontin (OPN/SPP1) isoforms collectively enhance tumor cell invasion and dissemination in esophageal adenocarcinoma. Oncotarget 6: 22239-22257, 2015.

9. Han DY, Fu D, Xi H, Li QY, Feng LJ, Zhang W, Ji G, Xiao JC and Wei Q: Genomic expression profiling and bioinformatics analysis of pancreatic cancer. Mol Med Rep 12: 4133-4140, 2015.

10. Zhang C, Peng L, Zhang Y, Liu Z, Li W, Chen S and Li G: The identification of key genes and pathways in hepatocellular carcinoma by bioinformatics analysis of high-throughput data. Med Oncol 34: 101, 2017.

11. Peng D, Guo Y, Chen H, Zhao S, Washington K, Hu T, Shyr Y and El-Rifai W: Integrated molecular analysis reveals complex interactions between genomic and epigenomic alterations in esophageal adenocarcinomas. Sci Rep 7: 40729, 2017.

12. Gentleman R: Bioinformatics and Computational Biology Solutions Using R and Bioconductor. Springer Science+Business Media, New York, 2005.

13. Carvalho B, Bengtsson H, Speed TP and Irizarry RA: Exploration, normalization, and genotype calls of high-density oligonucleotide SNP array data. Biostatistics 8: 485-499, 2007.

14. Smyth GK: Linear models and empirical bayes methods for assessing differential expression in microarray experiments. Stat Appl Genet Mol Biol 3: 1-25, 2004.

15. Bader GD and Hogue CW: An automated method for finding molecular complexes in large protein interaction networks. BMC Bioinformatics 4: 2, 2003.

16. Scardoni G, Tosadori G, Faizan M, Spoto F, Fabbri F and Laudanna C: Biological network analysis with CentiScaPe: Centralities and experimental dataset integration. F1000Res 3: 139, 2014.

17. Coleman HG, Xie SH and Lagergren J: The epidemiology of esophageal adenocarcinoma. Gastroenterology 154: 390-405, 2018.

18. Guglietta S and Rescigno M: Hypercoagulation and complement: Connected players in tumor development and metastases. Semin Immunol 28: 578-586, 2016

19. Alfaro C, Sanmamed MF, Rodríguez-Ruiz ME, Teijeira Á, Oñate C, González Á, Ponz M, Schalper KA, Pérez-Gracia JL and Melero I: Interleukin-8 in cancer pathogenesis, treatment and follow-up. Cancer Treat Rev 60: 24-31, 2017.

20. Li A, Varney ML, Valasek J, Godfrey M, Dave BJ and Singh RK: Autocrine role of interleukin-8 in induction of endothelial cell proliferation, survival, migration and MMP-2 production and angiogenesis. Angiogenesis 8: 63-71, 2005.

21. Shrivastava MS, Hussain Z, Giricz O, Shenoy N, Polineni R, Maitra A and Verma A: Targeting chemokine pathways in esophageal adenocarcinoma. Cell Cycle 13: 3320-3327, 2014.

22. Xiaoyun X, Chaofei H, Weiqi Z, Chen C, Lixia L, Queping L, Cong P, Shuang Z, Juan S and Xiang C: Possible involvement of F1F0-ATP synthase and intracellular ATP in keratinocyte differentiation in normal skin and skin lesions. Sci Rep 7: 42672, 2017.

23. Bernard BA, Asselineau D, Schaffar-Deshayes L and Darmon MY: Abnormal sequence of expression of differentiation markers in psoriatic epidermis: Inversion of two steps in the differentiation program? J Invest Dermatol 90: 801-805, 1988.

24. Shan J, Oshima T, Farre R, Fukui H, Watari J and Miwa H: IL-4 induces columnar-like differentiation of esophageal squamous epithelium through JAK/PI3K pathway: Possible role in pathogenesis of Barrett's esophagus. Am J Physiol Gastrointest Liver Physiol 306: G641-G649, 2014.

25. Jackson HW, Defamie V, Waterhouse P and Khokha R: TIMPs: Versatile extracellular regulators in cancer. Nat Rev Cancer 17: 38-53, 2017.

26. Kozłowski M, Laudański W, Mroczko B, Szmitkowski M, Milewski R and Łapuć G: Serum tissue inhibitor of metalloproteinase 1 (TIMP-1) and vascular endothelial growth factor A (VEGF-A) are associated with prognosis in esophageal cancer patients. Adv Med Sci 58: 227-234, 2013.

27. Tian L, Lu ZP, Cai BB, Zhao LT, Qian D, Xu QC, Wu PF, Zhu Y, Zhang JJ, Du Q, et al: Activation of pancreatic stellate cells involves an EMT-like process. Int J Oncol 48: 783-792, 2016. 
28. Pankov R and Yamada KM: Fibronectin at a glance. J Cell Sci 115: 3861-3863, 2002.

29. McMahon B and Kwaan HC: The plasminogen activator system and cancer. Pathophysiol Haemost Thromb 36: 184-194, 2008.

30. Placencio VR and DeClerck YA: Plasminogen activator inhibitor-1 in cancer: Rationale and insight for future therapeutic testing. Cancer Res 75: 2969-2974, 2015.

31. Jänicke F, Schmitt M and Graeff H: Clinical relevance of the urokinase-type and tissue-type plasminogen activators and of their type 1 inhibitor in breast cancer. Semin Thromb Hemost 17: 303-312, 1991.

32. Ortiz G, Salica JP, Chuluyan EH and Gallo JE: Diabetic retinopathy: Could the alpha-1 antitrypsin be a therapeutic option? Biol Res 47: 58, 2014

33. Li Y, Miao L, Yu M, Shi M, Wang Y, Yang J, Xiao Y and Cai $\mathrm{H}$ : $\alpha 1$-antitrypsin promotes lung adenocarcinoma metastasis through upregulating fibronectin expression. Int J Oncol 50 1955-1964, 2017.

34. Mutesa L, Azad AK, Verhaeghe C, Segers K, Vanbellinghen JF, Ngendahayo L, Rusingiza EK, Mutwa PR, Rulisa S, Koulischer L, et al: Genetic analysis of Rwandan patients with cystic fibrosis-like symptoms: Identification of novel cystic fibrosis transmembrane conductance regulator and epithelial sodium channel gene variants. Chest 135: 1233-1242, 2009.

35. Kerem B, Rommens JM, Buchanan JA, Markiewicz D, Cox TK, Chakravarti A, Buchwald M and Tsui LC: Identification of the cystic fibrosis gene: Genetic analysis. Science 245: 1073-1080, 1989.

36. Gharahkhani P, Fitzgerald RC, Vaughan TL, Palles C, Gockel I, Tomlinson I, Buas MF, May A, Gerges C, Anders M, et al; Barrett's and Esophageal Adenocarcinoma Consortium (BEACON); Esophageal Adenocarcinoma GenEtics Consortium (EAGLE); Wellcome Trust Case Control Consortium 2 (WTCCC2): Genome-wide association studies in oesophageal adenocarcinoma and Barrett's oesophagus: A large-scale meta-analysis. Lancet Oncol 17: 1363-1373, 2016.
37. Ward LD and Kellis M: HaploReg: A resource for exploring chromatin states, conservation, and regulatory motif alterations within sets of genetically linked variants. Nucleic Acids Res 40: D930-D934, 2012.

38. Rittling SR and Singh R: Osteopontin in immune-mediated diseases. J Dent Res 94: 1638-1645, 2015.

39. Zhang Z, Wang Y,Zhang J,Zhong J and Yang R: COL1A1 promotes metastasis in colorectal cancer by regulating the WNT/PCP pathway. Mol Med Rep 17: 5037-5042, 2018.

40. Tian ZQ, Li ZH, Wen SW, Zhang YF, Li Y, Cheng JG and Wang GY: Identification of commonly dysregulated genes in non-small-cell lung cancer by integrated analysis of microarray data and qRT-PCR validation. Hai 193: 583-592, 2015.

41. Song Y, Kim SH, Kim KM, Choi EK, Kim J and Seo HR: Activated hepatic stellate cells play pivotal roles in hepatocellular carcinoma cell chemoresistance and migration in multicellular tumor spheroids. Sci Rep 6: 36750, 2016.

42. Li J, Ding Y and Li A: Identification of COL1A1 and COL1A2 as candidate prognostic factors in gastric cancer. World J Surg Oncol 14: 297, 2016.

43. Casselbrant A, Edebo A, Wennerblom J, Lönroth H, Helander HF, Vieth M, Lundell L and Fändriks L: Actions by angiotensin II on esophageal contractility in humans. Gastroenterology 132: 249-260, 2007

44. Wang D and Dubois RN: Eicosanoids and cancer. Nat Rev Cancer 10: 181-193, 2010

This work is licensed under a Creative Commons Attribution-NonCommercial-NoDerivatives 4.0 International (CC BY-NC-ND 4.0) License. 